\section{References}

Beck, A. (1965). Mycobacterium fortuitum in abscesses of man. Journal of Clinical Pathology, 18, 307-313.

Hand, W. L., and Sanford, J. P. (1970). Mycobacterium fortuitum - a human pathogen. Annals of Internal Medicine, 73, 971-977.

Jenkins, P. A., and Marks, J. (1971). Transient colonisation of the respiratory tract by mycobacteria in South Wales. Tubercle, 52, 60-66.
Marks, J. (1976). A system for the examination of tubercle bacilli and other mycobacteria. Tubercle, 57, 207-225.

Marks, J., and Szulga, T. (1965). Thin-layer chromatography of mycobacterial lipids as an aid to classification. Technical procedures: Mycobacterium fortuitum. Tubercle, 46, 400411.

Correspondence to Dr J. R. Sibert, Department of Child Health, Llandough Hospital, Penarth, Glamorgan CF6 1XX.

\title{
Progressive hypogammaglobulinaemia in a child born to a mother with Hodgkin's disease
}

\author{
D. I. K. EVANS AND J. L. BURN
}

Departments of Child Health and Haematology, Royal Manchester Children's Hospital, and Bolton General Hospital, Lancashire

SUMMARY A young woman developed Hodgkin's disease (nodular sclerosis) in pregnancy and gave birth to a boy who developed common variable immunodeficiency. Initially there was normal IgG with low IgA and IgM, and antibody deficiency. IgG levels fell progressively over 4 years. Cellular immunity was normal. We suggest that this is a further family with immune deficiency presenting with common variable immunodeficiency and lymphoid malignancy.

The association of immunodeficiency with lymphoid malignancy has been reported. We report an unusual combination in a family: a young woman developed Hodgkin's disease in the 3rd trimester of pregnancy and gave birth to a boy who was found later to have common variable immunodeficiency. The father and elder child are normal.

\section{Case histories}

The mother. The mother's past medical history was uneventful until her appendix was removed for acute appendicitis at 28 years; during convalescence she developed infective hepatitis.

She noted enlarged cervical lymph nodes during her normal 2nd pregnancy at age 29. A month after confinement she developed a large liver and spleen. A lymph node biopsy showed Hodgkin's disease (nodular sclerosis). She also had lymphopenia $\left(0 \cdot 38 \times 10^{9} / 1\right)$. She was treated with radiotherapy and chemotherapy but died 4 years later after a generalised herpes zoster infection.
Lymphocyte counts during remission were often $<1.5 \times 10^{9} / 1$ and usually $<1.0 \times 10^{9} / 1$. No other tests of immunological function were done.

The child. Her son was born normally at term weighing $3 \cdot 23 \mathrm{~kg}$. He was bottle fed. Growth and development in infancy were normal. He received 3 doses of triple antigen (diphtheria/pertussis/tetanus) and oral polio vaccine during the 1st year of life, and a booster at 18 months. Since then he has had chicken pox and mumps. He has had recurrent nasal discharge, fever, nocturnal cough, occasional wheezing and exertional dyspnoea, intermittent diarrhoea, and two attacks of pneumonia, associated with neutrophil leucocytosis.

At age $5 \frac{1}{2}$, he developed herpes zoster. Apart from mild superficial scarring, there were no sequelae. Serum immunoglobulin concentrations were IgG $6 \cdot 0-6 \cdot 2 \mathrm{~g} / 1, \operatorname{IgA} 0 \cdot 12$ and $<0 \cdot 10 \mathrm{~g} / 1$. IgM was not detectable. Finger clubbing, a prominent sternum, and persistent coarse adventitial sounds over the right middle lobe were noted. Tonsils and adenoids were very small. He had no lymphadenopathy or enlargement of liver or spleen. $X$-ray showed chronic inflammatory changes in the right upper and lower lobes suggesting bronchiectasis.

At age 6 years, haemoglobin was 11.2 to 12.4 $\mathrm{g} / \mathrm{dl}$, with WBC 10.7 to $12.4 \times 10^{9} / 1$, neutrophils $62 \%$, lymphocytes $33 \%$, monocytes $3 \%$, eosinophils $2 \%$. The absolute lymphocyte count was approximately $4.0 \times 10^{9} / 1$, small lymphocytes $42 \%$, medium lymphocytes $44 \%$, large lymphocytes $14 \%$. Tests of immunity are shown in the Table.

The neutrophil NBT test showed $3 \%$ positive cells 
Table Immunological investigations at age 6 years

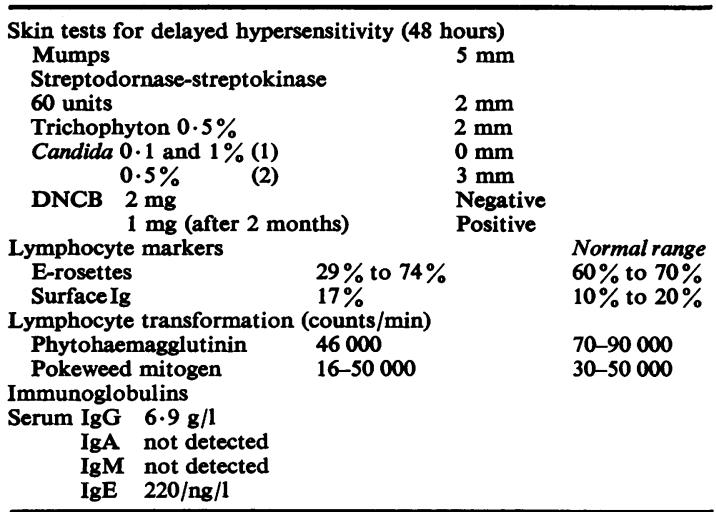

in the resting state, and $72 \%$ after latex phagocytosis.

The saliva contained IgG $0 \cdot 16 \mathrm{~g} / \mathrm{l}$ and $\operatorname{IgA} 0.5 \mathrm{~g} / \mathrm{l}$.

IgM was not present, but free secretory component was detected. The serum IgG showed a reversal of the normal 2:1 kappa to lambda ratio, and was short on immunoelectrophoresis. No antibodies to milk products were present. No antibody was detected to rubella, measles, cytomegalovirus, varicella, mumps, polio type 2 , or pertussis. Antinuclear factor was negative.

The blood group was $\mathrm{O} R \mathrm{R}$ (D) positive. No anti-A or anti-B was present. The HLA type was A1, A3, B5, B7. The direct antiglobulin test was negative. The bone marrow contained $30 \%$ lymphocytes, mainly small forms. No plasma cells were seen in over 1000 cells.

An inguinal node biopsy was taken after a booster dose of diphtheria/pertussis/tetanus into the anterior surface of the right thigh. The node was small and dissected with difficulty. There was little surrounding fibrosis. Cortical lymphoid tissue was sparse with very poor follicle formation and no reacting centres. The paracortical area was normally cellular. Macrophages were present in the peripheral sinuses.

Total haemolytic complement was normal, with normal $\mathrm{C} 1$ inhibitor and $\mathrm{Clq}$. C3 was $0.85 \mathrm{~g} / 1$ and $\mathrm{C} 40.3 \mathrm{~g} / 1$. No virus was isolated from faeces, but adenovirus type 7 was isolated from a sputum sample on one occasion. Bacterial cultures of sputum grew Haemophilus influenzae. On a fat intake of $50 \mathrm{~g} /$ day for 3 days the faeces contained $4 \cdot 5 \mathrm{~g}$ fat, i.e. $97 \%$ absorption.

Regular postural drainage and injections of immunoglobulin were started.

After a loading dose of 5 days' gammaglobulin, the serum IgG levels rose by the expected amount to $8.5 \mathrm{~g} / \mathrm{l}$, but on weekly maintenance, levels have progressively declined by approximately $1.0 \mathrm{~g} / 1$ per year and have remained at $3 \cdot 0-4 \cdot 0 \mathrm{~g} / 1$ during the last year with continued maintenance. IgA and IgM have not shown any significant changes. IgG was present in the saliva at first, but later became undetectable. There has been no change in the salivary IgA deficiency.

During 4 years of observation, sputum production, chest rales, and finger clubbing have regressed considerably. The chest $x$-ray is now normal. There has been intermittent alopecia areata, and a traumatic synovitis of the hip.

$\mathrm{He}$ is growing normally and doing well at school.

\section{Discussion}

The patient's immunodeficiency, with normal IgG, deficient $\operatorname{Ig} \mathrm{A}$ and IgM, and antibody deficiency, was at first similar to the case of Giedion and Scheidegger (1957), but without the profound defect of cellular immunity. However, IgG levels fell progressively during treatment with gammaglobulin, and salivary IgG also became undetectable. Skin tests showed positive but weak delayed hypersensitivity, and no conclusive defect of cellular immunity could be shown. B-cells were normal or raised, whereas in sex-linked gammaglobulinaemia they are usually reduced (Cooper and Lawton, 1972). Such patients are now grouped under common variable immunodeficiency. Although the progressive fall of $\operatorname{IgG}$ is unusual, it might be found in a larger number of cases if investigations were made early enough. An alternative explanation is that this IgG without detectable antibody activity has been suppressed by replacement with normal antibody.

The alopecia was attributed to trichitillomania, as he has been observed to pull his hair; but it may be a further example of the alopecia associated with hypogammaglobulinaemia described by Ipp and Gelfand (1976).

The mother's disease was histologically nodular sclerosis, the common type of Hodgkin's disease in young women. Lymphopenia is a common feature of Hodgkin's disease. However, Hodgkin's disease in pregnancy is not normally associated with illness in the baby. Poliwoda et al. (1967), in an extensive review, reported that 151 of 162 children born to mothers with Hodgkin's disease were normal. Six were stillborn and 10 died within a few hours or before they were three. Follow-up of such children has concentrated on the development of Hodgkin's disease, and not on immunodeficiency. However, these authors noted that one mother had two boys who both died, one with pneumonia and the other with obscure pyrexia and collapse, which could indicate a familial defect of immunity. 
We do not attribute our patient's illness to his mother's illness, but there is a well-known association between immunological abnormality and malignancy. Several families have been described with a high incidence of Hodgkin's disease or lymphoma and immunological defect (Potolsky et al., 1971; Buehler et al., 1975). There must be a genetic basis for this combination of disorders in some families, although the occurrence of Hodgkin's disease in husband and wife (Mazar and Straus, 1951) suggests an environmental cause in others. In our case we believe that one genetic defect of immunity has predisposed to malignancy and immunological deficiency.

We thank Dr R. A. Thompson for immunoglobulin estimations and for supplying replacement gammaglobulin, and Dr S. Kumar for tests of lymphocyte markers. Miss V. Rawlinson kindly measured serum complement.

\section{References}

Buehler, S. K., Firme, F., Fodor, G., Fraser, G. R., Marshall, W. H., and Vaze, P. (1975). Common variable immune deficiency, Hodgkin's disease, and other malignancies in a Newfoundland family. Lancet, 1, 195-197.

Cooper, M. D., and Lawton, A. R. (1972). Circulating Bcells in patients with immunodeficiency. American Journal of Pathology, 69, 513-528.

Giedion, A., and Scheidegger, J. J. (1957). Kongenitale Immunoparese bei Fehlen spezifischer $\beta 2$-Globuline und quantitativ normale $\gamma$-Globulinen. Helvetica paediatrica acta, 12, 241-259.

Ipp, M. M., and Gelfand, E. W. (1976). Antibody deficiency and alopecia. Journal of Pediatrics, 89, 728-731.

Mazar, S. A., and Straus, B. (1951). Marital Hodgkin's disease. Archives of Internal Medicine, 88, 819-830.

Poliwoda, H., Stolte, H., Voth, H., Gothe, H. D., and Kostering, H. (1967). Lymphogranulomatose und Schwangerschaft. Archiv für klinische Medizin, 213, 255-277.

Potolsky, A. I., Heath, C. W., Jr, Buckley, C. E., III, and Rowlands, D. T. (1971). Lymphoreticular malignancies and immunologic abnormalities in a sibship. American Journal of Medicine, 50, 42-48.

Correspondence to Dr D. I. K. Evans, Royal Manchester Children's Hospital, Pendlebury, Manchester M27 1HA.

\title{
Intrapartum sensitisation of human infants to the Rhesus-D antigen
}

\author{
COLIN M. STERN \\ Department of Paediatrics and Neonatal Medicine, Hammersmith Hospital
}

SUMMARY A study is reported of the numbers of nonimmunoglobulin-bearing lymphocytes specifically binding to Rhesus-D positive erythrocytes in the blood of Rhesus-dd infants born to either Rhesus-dd or Rhesus-Dd mothers, at birth and at 6 months of age. There was a significant increase in the number of such antigen-binding cells by age 6 months in babies born to Rhesus-Dd mothers. It is concluded that the Rhesus-negative offspring of Rhesuspositive mothers may be sensitised against the Rhesus-D antigen at birth and that protection of such female infants by the injection of antiRhesus-D antibody should be considered.

The human infant at birth is capable of humoral antibody production against specific antigens. Although these responses are almost confined to immunoglobulin $\mathbf{M}$ (IgM) production, small amounts of immunoglobulin $\mathrm{G}$ ( $\mathrm{IgG}$ ) can be detected. Immune responses to most antigens require $T$ lymphocyte help and therefore the presence of specifically reacting $\mathrm{T}$-lymphocytes. The response to the Rhesus-D (Rh-D) antigen is thought to be of this kind.

Since the introduction of treatment with pooled antiRh-D antiserum for Rh-dd mothers postnatally, the incidence of haemolytic disease of the newborn has fallen dramatically. A few new patients are still found, usually ascribed either to the injection or infusion of Rh-incompatible blood or to a failure in treatment with antiRh-D antiserum (Woodrow, 1974). Some studies have suggested, intriguingly, that the birth of a Rh-dd infant to a Rh-Dd mother could lead to intrapartum sensitisation of the offspring, whose latent sensitivity might be reactivated many years later, during the gestation of a Rh-Dpositive fetus. Ramos de Almeida and Rosado (1972) found that the incidence of rhesus isoimmunisation by birth rank was dependent upon the grandmaternal Rh blood group, while Taylor (1967) and Hindemann (1973) suggest that sensitisation against Rh-D in infancy may be a significant event.

Some Rh-dd male infants, the offspring of either Rh-dd or Rh-Dd mothers, were selected by the Rh blood group and the ability of their peripheral blood 